\title{
Frequency domain structural synthesis applied to quasi-static crack growth modeling
}

\author{
Young W. Kwon* and Joshua H. Gordis \\ Department of Mechanical \& Astronautical Engineering, Naval Postgraduate School, Monterey, CA 93943, USA
}

Received 4 September 2008

Revised January 2009

\begin{abstract}
Quasi-static crack growth in a composite beam was modeled using the structural synthesis technique along with a finite element model. The considered crack was an interface crack in the shear mode (i.e. mode II), which occurs frequently in the scarf joint of composite structures. The analysis model was a composite beam with an edge crack at the midplane of the beam subjected to a three-point bending load. In the finite element model, beam finite elements with translational degrees of freedom only were used to model the crack conveniently. Then, frequency domain structural synthesis (substructure coupling) was applied to reduce the computational time associated with a repeated finite element calculation with crack growth. The quasi-static interface crack growth in a composite beam was predicted using the developed computational technique, and its result was compared to experimental data. The computational and experimental results agree well. In addition, the substructure-based synthesis technique showed the significantly improved computational efficiency when compared to the conventional full analysis.
\end{abstract}

Keywords: Structural synthesis, substructure, finite element, crack growth, composite

\section{Introduction}

The failure analysis of large-scale structural systems such as aircraft and ships is a computationally demanding task, and hence is frequently pursued on a component basis. At a basic level, the computational demand is due to the need for a refined mesh surrounding the crack area, while maintaining a mesh of adequate refinement for the entire structure. The computational demand is significantly increased by the presence of composite materials due to the need to include through-the-thickness computations for ply failure. The computational demand is further increased when nonlinear structural behavior is included. In this paper, we explore the use of substructuring techniques in the failure analysis of structural systems.

Substructure coupling, or substructuring refers to the analysis of a structural system by the joining together of substructures, each of which is analyzed independently. The resulting assemblage of substructures is typically a system model of significantly fewer degrees-of-freedom (DOF) than the total number of DOF, hence the computational advantage of a substructuring procedure. The most well-known of the substructuring procedures are known as component mode synthesis procedures. The substructures are each represented by sets of shapes associated with a combination of physical and modal coordinates. A distinctive feature of component mode synthesis procedures is that the substructure representation requires mode shape information for all DOF for each substructure. Additionally, the system model size grows as additional substructure modes are added to increase the accuracy of the system model.

In contrast to component mode synthesis, here we will make use of a coupling procedure which is distinguished by its functioning solely in physical coordinates, and which requires the retention of coupling DOF only from

*Corresponding author. E-mail: ywkwon@nps.edu. 


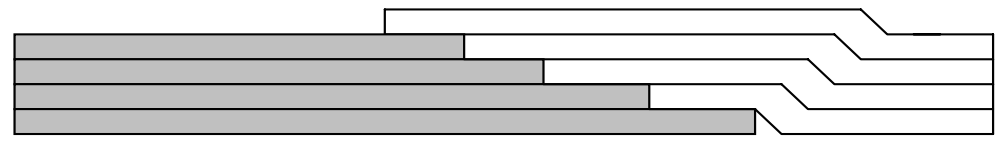

Fig. 1. Scarf joint of two composites, one in gray and the other in white.

each of the substructures. A significant literature exists on this topic; see for example [1-7]. A frequency domain formulation [6,7] which was adopted in the present work, represents substructures by frequency response functions (FRF) calculated at the connect DOF. Using the FRF of the substructures, which need be calculated only once, the coupled system frequency response can be directly synthesized. Additionally, the coupling forces which act on the substructures can be calculated. What distinguishes both the time and frequency domain coupling methods is that the substructures are represented only by response functions at the coupling DOF only - no internal DOF (non-coupling DOF which are "internal" to the substructures) need be retained.

Here we will develop a hybrid frequency domain substructure/crack growth analysis methodology for an interface crack in a composite structure. We exploit the property of the frequency domain formulation wherein each substructure is represented only by coupling DOF. This will allow those portions of the structures not containing a crack to be eliminated from the analysis, except for the coupling DOF. Note that as the frequency domain formulation is exact, no approximation is introduced, regardless of the size and number of the substructures whose internal (noncoupling) DOF are eliminated. We will demonstrate the computational advantage of the hybrid frequency domain substructure/crack growth analysis methodology, as compared with a traditional full-up analysis of the damaged structure.

Interface crack failure is common in composites structures, especially in scarf joints of composite structures [8] as sketched in Fig. 1. In the scarf joint, cracks initiate at interfaces of two adjacent layers of the joint section and propagate to complete failure. In particular, the critical crack forms at the high stress concentration location, which is in most cases at the bottom interface of the joint of Fig. 1. In order to predict the failure strength of the scarf joint, it is necessary to be able to model interface crack propagation in composites. As a result, the present study considers modeling of a quasi-static interface crack growth in a composite beam.

The subsequent section describes the frequency domain substructuring technique, which is followed by the finite element modeling for crack growth. Then, an example problem is provided for quasi-static crack growth in a composite beam. For this example, both computer modeling results (traditional full-up analysis and the substructuring methodology) are compared to the experimental data. In addition, the substructuring synthesis model is compared to the conventional full analysis model so as to determine the relative computational time. Finally, conclusions are provided at the end.

\section{Frequency domain substructuring}

Frequency domain synthesis is a general formulation for structural modification and substructure coupling [6, 7]. Using frequency response functions (FRF) to represent the substructures, the formulation is exact and as a minimum, requires only FRF at the coupling DOF. The generation of FRF is done once, and the full model need not be resolved subsequently. The frequency response functions are defined quite generally, and may include stress and strain frequency response as needed, e.g.:

$$
H_{i j}(\Omega) \equiv \frac{\partial x_{i}(\Omega)}{\partial f_{j}(\Omega)} \quad H_{i j}^{\sigma}(\Omega) \equiv \frac{\partial \sigma_{i}(\Omega)}{\partial f_{j}(\Omega)} \quad H_{i j}^{\varepsilon}(\Omega) \equiv \frac{\partial \varepsilon_{i}(\Omega)}{\partial f_{j}(\Omega)}
$$

where Eq. (1) denote frequency response functions which relate displacement, stress, and strain response at DOF “ $\mathrm{i}$ ” to a force at DOF “j”, respectively.

We will summarize the principal elements of the formulation which are pertinent to the coupling problem at hand. We will be applying the procedure to a quasi-static analysis, a prior example of which can be found in [9]. A key aspect of the application to static problems is the need to install springs to ground in the substructure models prior to synthesis (due to the need to form flexibility matrices) and the subsequent removal of these springs in the synthesis, concurrently with the coupling. 

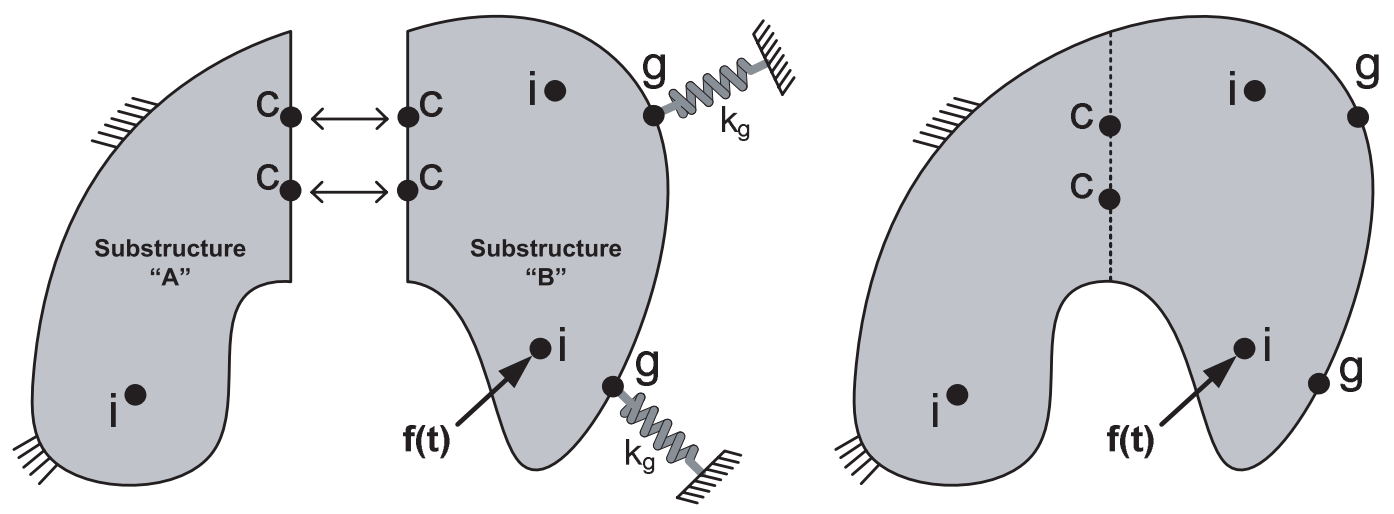

Fig. 2. Uncoupled and coupled substructures.

Referring to Fig. 2, two substructures, denoted "A" and "B" are shown. For each substructure, a set of coupling DOF are identified, and are members of the "c" set of DOF. Those DOF not directly involved in coupling, but for which synthesized (i.e. coupled) responses are desired are included as needed in the "i" set. Those DOF at which synthesized stress response is desired are members of the " $\sigma$ " set. For those substructures with rigid-body modes requiring the installation of springs-to-ground prior to the calculation of uncoupled flexibilities (zero-frequency response), the DOF at which the springs-to-ground are installed are members of the "g" set.

The steady-state equation of motion in the frequency domain is given by Eq. (2). Note that vector/matrix quantities are in bold text. Note that the various partitions of the matrix in this equation contain entries from all substructures. In other words, each DOF set may have members from all substructures.

$$
\left\{\begin{array}{c}
\boldsymbol{\sigma} \\
\mathbf{x}_{i} \\
\mathbf{x}_{c} \\
\mathbf{x}_{g}
\end{array}\right\}=\left[\begin{array}{lll}
\mathbf{H}_{\sigma i} & \mathbf{H}_{\sigma c} & \mathbf{H}_{\sigma g} \\
\mathbf{H}_{i i} & \mathbf{H}_{i c} & \mathbf{H}_{i g} \\
\mathbf{H}_{c i} & \mathbf{H}_{c c} & \mathbf{H}_{c g} \\
\mathbf{H}_{g i} & \mathbf{H}_{g c} & \mathbf{H}_{g g}
\end{array}\right]\left\{\begin{array}{c}
\mathbf{f}_{i} \\
\mathbf{f}_{c} \\
\mathbf{f}_{g}
\end{array}\right\}
$$

From the definition of the DOF sets (c-set, i-set, $\sigma$-set, g-set), the loading at the member DOF is comprised of externally applied loads, and in all cases except the i-set, loads due to coupling, i.e.

$$
\left\{\begin{array}{l}
\mathbf{f}_{e} \\
\mathbf{f}_{c} \\
\mathbf{f}_{g}
\end{array}\right\}=\left\{\begin{array}{l}
\mathbf{f}_{e} \\
\mathbf{f}_{c} \\
\mathbf{f}_{g}
\end{array}\right\}^{e}+\left\{\begin{array}{c}
0 \\
\mathbf{f}_{c} \\
\mathbf{f}_{g}
\end{array}\right\}^{*}
$$

where the "e" superscript indicates externally applied loads, and the * superscript indicates a coupled (synthesized) quantity.

Making use of Eq. (3) along with the set union,

$$
e=i \cup c \cup g
$$

we can rewrite Eq. (2) as,

$$
\left\{\begin{array}{c}
\boldsymbol{\sigma} \\
\mathbf{x}_{e} \\
\mathbf{x}_{c} \\
\mathbf{x}_{g}
\end{array}\right\}=\left[\begin{array}{lll}
\mathbf{H}_{\sigma e} & \mathbf{H}_{\sigma c} & \mathbf{H}_{\sigma g} \\
\mathbf{H}_{e e} & \mathbf{H}_{e c} & \mathbf{H}_{e g} \\
\mathbf{H}_{c e} & \mathbf{H}_{c c} & \mathbf{H}_{c g} \\
\mathbf{H}_{g e} & \mathbf{H}_{g c} & \mathbf{H}_{g g}
\end{array}\right]\left\{\begin{array}{l}
\mathbf{f}_{e} \\
\mathbf{f}_{c} \\
\mathbf{f}_{g}
\end{array}\right\}
$$

which now includes redundant rows, since $c \subset e$.

As explained in Ref. [9], the coupling connectivity to be established between the c-set and g-set DOF is represented by a Boolean mapping matrix, $\left[\mathbf{M}_{c}\right]$, whose rows correspond to the c-set DOF and the columns correspond to the connections. For example, for a single pair of c-set coordinates to be coupled, $\left[\mathbf{M}_{c}\right]$ is

$$
\left[\mathbf{M}_{c}\right]=\left[\begin{array}{r}
1 \\
-1
\end{array}\right]
$$


This matrix establishes the "pairing" among the c-set coordinates. For a spring-to-ground (one end restrained), the mapping matrix is simply

$$
\left[\mathbf{M}_{g}\right]=[1]
$$

where the pairing is between g-set coordinates on the substructures and (non-existent) ground coordinates, hence the missing lower row in $\left[\mathbf{M}_{g}\right]$.

In Ref. [6,7,9], it is shown that the mapping matrices are related to directed graphs, which can be used to graphically portray the synthesis, and can also be found from equilibrium of the connection. The mapping matrices are associated with "reduced" impedances for an interconnecting impedance, and the reduced impedance $\tilde{Z}$ for single spring-to-ground is,

$$
\tilde{\mathbf{Z}}=k_{g}
$$

The mapping matrices serve to define the reduced coordinates. For the c-set coordinates, we have

$$
\begin{aligned}
& \left\{\mathbf{f}_{c}\right\}=\left[\mathbf{M}_{c}\right]\left\{\tilde{\mathbf{f}}_{c}\right\} \\
& \left\{\tilde{\mathbf{x}}_{c}\right\}=\left[\mathbf{M}_{c}\right]^{T}\left\{\mathbf{x}_{c}\right\}
\end{aligned}
$$

For the complete development of these results, see Ref. [6,7,9].

We now construct the synthesis transformations which will operate on Eq. (4).

$$
\begin{gathered}
\left\{\begin{array}{c}
\boldsymbol{\sigma} \\
\mathbf{x}_{e} \\
\tilde{\mathbf{x}}_{c} \\
\tilde{\mathbf{x}}_{g}
\end{array}\right\}=\left[\begin{array}{cccc}
\mathbf{I} & 0 & 0 & 0 \\
0 & \mathbf{I} & 0 & 0 \\
0 & 0 & \mathbf{M}_{c}^{T} & 0 \\
0 & 0 & 0 & \mathbf{M}_{g}^{T}
\end{array}\right]\left\{\begin{array}{c}
\sigma \\
\mathbf{x}_{e} \\
\mathbf{x}_{c} \\
\mathbf{x}_{g}
\end{array}\right\} \\
\left\{\begin{array}{c}
\mathbf{f}_{e} \\
\mathbf{f}_{c} \\
\mathbf{f}_{g}
\end{array}\right\}=\left[\begin{array}{ccc}
\mathbf{I} & 0 & 0 \\
0 & \mathbf{M}_{c} & 0 \\
0 & 0 & -\mathbf{M}_{g} \tilde{Z}
\end{array}\right]\left\{\begin{array}{c}
\mathbf{f}_{e} \\
\tilde{\mathbf{f}}_{c} \\
\tilde{\mathbf{x}}_{g}
\end{array}\right\}
\end{gathered}
$$

Using these transformations, Eq. (4) becomes

$$
\left\{\begin{array}{c}
\boldsymbol{\sigma} \\
\mathbf{x}_{e} \\
\tilde{\mathbf{x}}_{c} \\
\tilde{\mathbf{x}}_{g}
\end{array}\right\}=\left[\begin{array}{ccc}
\mathbf{H}_{\sigma e} & \mathbf{H}_{\sigma c} \mathbf{M}_{c} & -\mathbf{H}_{\sigma g} \mathbf{M}_{g} \tilde{\mathbf{Z}} \\
\mathbf{H}_{e e} & \mathbf{H}_{e c} \mathbf{M}_{c} & -\mathbf{H}_{e g} \mathbf{M}_{g} \tilde{\mathbf{Z}} \\
\mathbf{M}_{c}^{T} H_{c e} & \mathbf{M}_{c}^{T} \mathbf{H}_{c c} \mathbf{M}_{c} & -\mathbf{M}_{c}^{T} \mathbf{H}_{c g} \mathbf{M}_{g} \tilde{\mathbf{Z}} \\
\mathbf{M}_{g}^{T} \mathbf{H}_{g e} & \mathbf{M}_{g}^{T} \mathbf{H}_{g c} \mathbf{M}_{c} & -\mathbf{M}_{g}^{T} \mathbf{H}_{g g} \mathbf{M}_{g} \tilde{\mathbf{Z}}
\end{array}\right]\left\{\begin{array}{c}
\mathbf{f}_{e} \\
\tilde{\mathbf{f}}_{c} \\
\tilde{\mathbf{x}}_{g}
\end{array}\right\}
$$

Extracting the third and fourth rows of Eq. (9)

$$
\left\{\begin{array}{c}
\tilde{\mathbf{x}}_{c} \\
\tilde{\mathbf{x}}_{g}
\end{array}\right\}=\left[\begin{array}{c}
\mathbf{M}_{c}^{T} \mathbf{H}_{c e} \\
\mathbf{M}_{g}^{T} \mathbf{H}_{g e}
\end{array}\right]\left\{\mathbf{f}_{e}\right\}+\left[\begin{array}{ll}
\mathbf{M}_{c}^{T} \mathbf{H}_{c c} \mathbf{M}_{c} & -\mathbf{M}_{c}^{T} \mathbf{H}_{c g} \mathbf{M}_{g} \tilde{\mathbf{Z}} \\
\mathbf{M}_{g}^{T} \mathbf{H}_{g c} \mathbf{M}_{c} & -\mathbf{M}_{g}^{T} \mathbf{H}_{g g} \mathbf{M}_{g} \tilde{\mathbf{Z}}
\end{array}\right]\left\{\begin{array}{c}
\tilde{\mathbf{f}}_{c} \\
\tilde{\mathbf{x}}_{g}
\end{array}\right\}
$$

Imposing compatibility on the c-set coordinates,

$$
\left\{\tilde{x}_{c}\right\}=\{0\}
$$

leads to,

$$
\left\{\begin{array}{c}
\tilde{\mathbf{f}}_{c} \\
\tilde{\mathbf{x}}_{g}
\end{array}\right\}=\left[\begin{array}{cc}
-\mathbf{M}_{c}^{T} \mathbf{H}_{c c} \mathbf{M}_{c} & \mathbf{M}_{c}^{T} \mathbf{H}_{c g} \mathbf{M}_{g} \tilde{\mathbf{Z}} \\
-\mathbf{M}_{g}^{T} \mathbf{H}_{g c} \mathbf{M}_{c} & \mathbf{I}+\mathbf{M}_{g}^{T} \mathbf{H}_{g g} \mathbf{M}_{g} \tilde{\mathbf{Z}}
\end{array}\right]^{-1}\left[\begin{array}{c}
\mathbf{M}_{c}^{T} \mathbf{H}_{c e} \\
\mathbf{M}_{g}^{T} \mathbf{H}_{g e}
\end{array}\right]\left\{\mathbf{f}_{e}\right\}
$$

Using Eq. (11) in the first two rows of Eq. (9) yields,

$$
\left\{\begin{array}{c}
\boldsymbol{\sigma} \\
\mathbf{x}_{e}
\end{array}\right\}=\left[\begin{array}{c}
\mathbf{H}_{\sigma e} \\
\mathbf{H}_{e e}
\end{array}\right]\left\{\mathbf{f}_{e}\right\}+\left[\begin{array}{l}
\mathbf{H}_{\sigma c} \mathbf{M}_{c}-\mathbf{H}_{\sigma g} \mathbf{M}_{g} \tilde{\mathbf{Z}} \\
\mathbf{H}_{e c} \mathbf{M}_{c}-\mathbf{H}_{e g} \mathbf{M}_{g} \tilde{\mathbf{Z}}
\end{array}\right]\left[\begin{array}{l}
-\mathbf{M}_{c}^{T} \mathbf{H}_{c c} \mathbf{M}_{c} \quad \mathbf{M}_{c}^{T} \mathbf{H}_{c g} \mathbf{M}_{g} \tilde{Z} \\
-\mathbf{M}_{g}^{T} \mathbf{H}_{g c} \mathbf{M}_{c} \mathbf{I}+\mathbf{M}_{g}^{T} \mathbf{H}_{g g} \mathbf{M}_{g} \tilde{Z}
\end{array}\right]^{-1}\left[\begin{array}{l}
\mathbf{M}_{c}^{T} \mathbf{H}_{c e} \\
\mathbf{M}_{g}^{T} \mathbf{H}_{g e}
\end{array}\right]\left\{\mathbf{f}_{e}\right\}
$$

Recognizing the new relationship between the applied loads and the $\sigma$-set and e-set responses yields the operative form synthesis transformation:

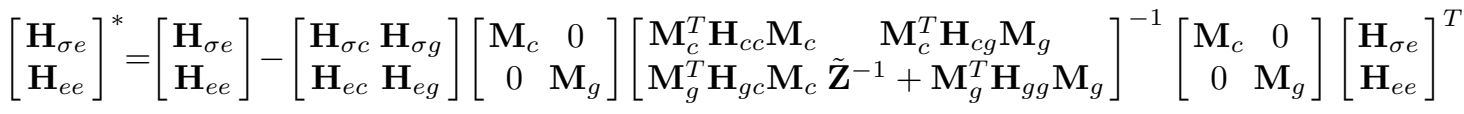

Equation (13) provides for the simultaneous coupling of substructure and removal of grounding springs. 


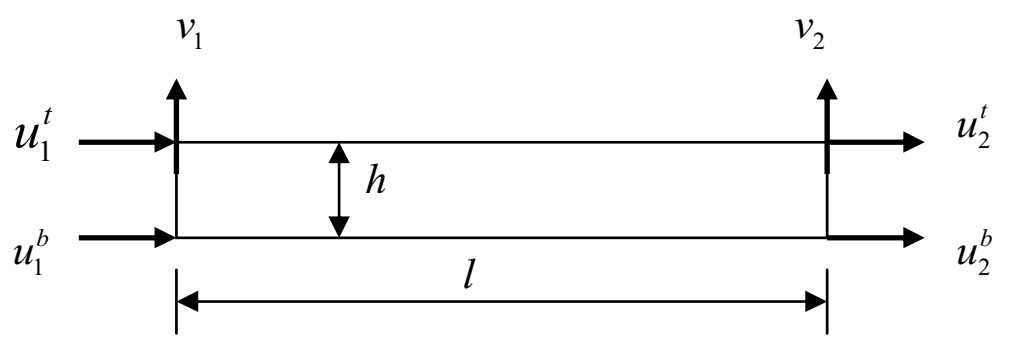

Fig. 3. Displacement-based beam element.

\section{Finite element modeling for crack growth}

Shearing mode crack growth, so called Mode II, was modeled for a composite beam joining interface strength. To this end, the displacement-based beam element was used to accommodate the crack and its growth conveniently [10, 11]. The beam element has both in-plane and transverse displacement degrees of freedom as sketched in Fig. 3, but no rotational degree of freedom. In addition, the transverse displacement is assumed to be constant through the beam thickness because the transverse normal strain is considered negligible. As a result, there are three degrees of freedom (i.e. two in-plane displacements and one transverse displacement) per node and six degrees of freedoms per beam element if the element has two nodes as shown in Fig. 3.

The stiffness matrix for the beam element is developed below. The in-plane displacement field of the element is expressed as

$$
u(x, y)=\sum_{i=1}^{n} N_{i}(x)\left[H_{1}(y) u_{i}^{b}+H_{2}(y) u_{i}^{t}\right]
$$

and the transverse displacement field is given by

$$
v(x)=\sum_{i=1}^{n} N_{i}(x) v_{i}
$$

in which superscripts ' $b$ ' and ' $t$ ' denote bottom and top sides of the beam, and $n=2$ for the beam element shown in Fig. 3. The shape functions are expressed as

$$
\begin{aligned}
& N_{1}(x)=1-\frac{x}{l} \\
& N_{2}(x)=\frac{x}{l} \\
& H_{1}(y)=1-\frac{y}{h} \\
& H_{2}(y)=\frac{y}{h}
\end{aligned}
$$

Here, $l$ and $h$ are the length and height of the beam element, respectively.

Normal and shear strains resulting from the displacement fields are computed as

$$
\varepsilon_{x}=\frac{\partial u}{\partial x}=\sum_{i=1}^{2} \frac{\partial N_{1}}{\partial x}\left[H_{1} u_{i}^{b}+H_{1} u_{i}^{b}\right]=\left\{B_{b}\right\}^{T}\{d\}
$$

and

$$
\gamma_{x y}=\frac{\partial u}{\partial y}+\frac{\partial v}{\partial x}=\sum_{i=1}^{2}\left(N_{i}\left[\frac{\partial H_{1}}{\partial y} u_{i}^{b}+\frac{\partial H_{2}}{\partial y} u_{i}^{b}\right]+\frac{\partial N_{i}}{\partial x} v_{i}\right)=\left\{B_{s}\right\}^{T}\{d\}
$$




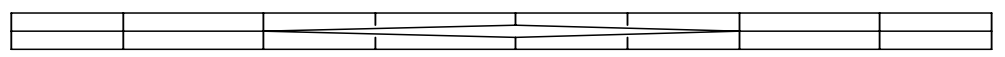

Fig. 4. Beam containing a crack along its longitudinal direction

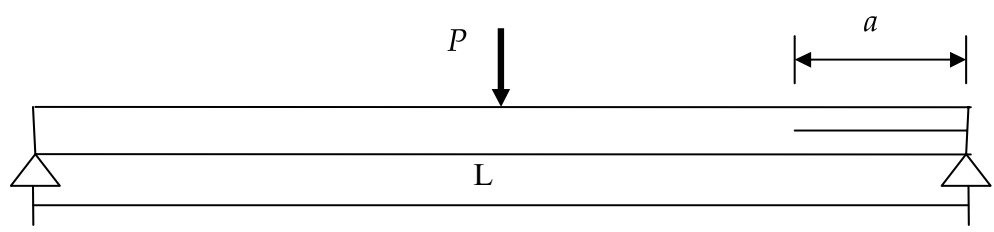

Fig. 5. Three point bending test for Mode II fracture.

where the elemental nodal displacement vector is given by

$$
\{d\}^{T}=\left\{u_{1}^{b} u_{1}^{t} v_{1} u_{2}^{b} u_{2}^{t} v_{2}\right\}
$$

Then, the element stiffness matrix is computed by summing bending stiffness and transverse shear stiffness as shown below:

$$
\left[K^{e}\right]=\left[K_{b}^{e}\right]+\left[K_{s}^{e}\right]
$$

where the bending stiffness matrix is

$$
\left[K_{b}^{e}\right]=\int_{0}^{l} \int_{0}^{h}\left\{B_{b}\right\} E\left\{B_{b}\right\}^{T} d y d x
$$

and the transverse shear stiffness matrix is

$$
\left[K_{s}^{e}\right]=\int_{0}^{l} \int_{0}^{h}\left\{B_{s}\right\} G\left\{B_{s}\right\}^{T} d y d x
$$

in which $E$ and $G$ are elastic and shear moduli, respectively. Finally, the element stiffness matrix is given by

$$
\left[K^{e}\right]=\left[\begin{array}{cccccc}
a_{1}+2 a_{3} & -a_{1}+a_{3} & a_{4} & a_{1}-2 a_{3} & -a_{1}-a_{3}-a_{4} \\
-a_{1}+a_{3} & a_{1}+2 a_{3} & -a_{4} & -a_{1}-a_{3} & a_{1}-2 a_{3} & a_{4} \\
a_{4} & -a_{4} & a_{2} & a_{4} & -a_{4} & -a_{2} \\
a_{1}-2 a_{3} & -a_{1}-a_{3} & a_{4} & a_{1}+2 a_{3} & -a_{1}+a_{3} & -a_{4} \\
-a_{1}-a_{3} & a_{1}-2 a_{3} & -a_{4} & -a_{1}+a_{3} & a_{1}+2 a_{3} & a_{4} \\
-a_{4} & a_{4} & -a_{2} & -a_{4} & a_{4} & a_{2}
\end{array}\right]
$$

in which

$$
a_{1}=\frac{G l}{4 h}, a_{2}=\frac{G h}{l}, a_{3}=\frac{E h}{6 l}, a_{4}=\frac{G}{2}
$$

In order to model a crack along the beam axis, two beam elements were overlaid along the beam as sketched in Fig. 4. Because the beam element has only displacement degrees of freedom like a 2-D plane stress element, element interface between the top and bottom elements are natural. Likewise the crack faces can be easily modeled with displacement degrees of freedom. As the crack grows, there should be a separation between top and bottom elements which are located along the path of crack growth. Therefore, top and bottom elements were considered to be connected by linear springs as constraints. Then, when the crack passes through the element, the springs are removed so that there is no need for remeshing for crack growth.

\section{Example problem}

The example problem selected was a three-point bending test of a carbon fiber composite beam with a crack at the midplane along the beam axis as sketched in Fig. 5. The test specimens were fabricated in two steps in order to 


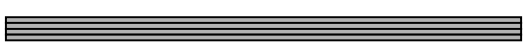

(a)

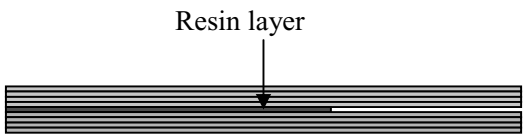

(c)

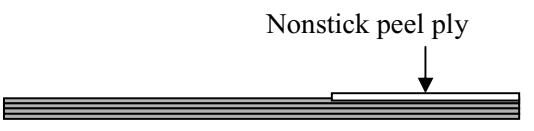

(b)

layers on the top of the bottom composite and cure them.

represent the joining interface of a composite. The bottom half of a composite beam was cured first with layers of composite fabrics using a resin. Then, top half composite layers were placed on the already cured composite beam, and they were cured with a resin. In order to create an initial crack, a non-sticking Teflon tape was placed partially between the top and bottom parts of the composite, as sketched in Fig. 6 . The main reason that the whole specimens were not cured together simultaneously was to represent the joining interface as in the composite scarf joint where a new composite part is added and cured on top of an already constructed and cured composite structure.

The physical test was conducted to measure the energy release of Mode II fracture of the joint interface of composite beams which was $128 \mathrm{~mm}$ long, $4.2 \mathrm{~mm}$ high, and $25.1 \mathrm{~mm}$ wide. Elastic and shear moduli of the beam were $50.4 \mathrm{GPa}$, and $3.17 \mathrm{GPa}$, respectively. The initial crack at the midplane of the beam was $50 \mathrm{~mm}$. The Mode II energy release rate, $\mathrm{G}_{I I}$, was determined using a compliance approach with the following equation [12]:

$$
G_{I I}=\frac{9 a^{2} P^{2} C}{2 b\left(2 L^{3}+3 a^{3}\right)}
$$

where:

$\mathrm{P}=$ applied load

$\mathrm{C}=$ compliance $(=$ deflection/force)

$a=$ initial delamination length

$b=$ sample width

$L=$ span length

The critical energy release rate $\mathrm{G}_{I I c}$ is computed with the applied load at the onset of crack propagation as the applied load increases very slowly. The unit of $\mathrm{G}_{I I c}$ is force/length. As the crack grows, the overall beam stiffness becomes less. Therefore, the load vs. displacement relationship at the center of the beam becomes nonlinear along with the crack growth. This experimental data is compared to the computational results later.

During the physical experiment, the displacement was controlled by increasing it very slowly to represent a quasi-static crack growth, for which the effect of kinetic energy is neglected. In order to simulate the experiment in the computer model, the displacement was applied incrementally at the beam center to determine the resulting force. Then, the energy release rate of Mode II is computed from Eq. (28) using the compliance of the force-displacement data. If the computed energy release rate is greater than the critical energy release rate, the crack is considered to grow until the new calculated energy release rate becomes less than the critical value. Because of the displacement control, the load and the energy release rate decrease under a constant displacement condition as the crack grows.

In order to model the present problem using the synthesis technique, the beam was constructed by two substructures as shown in Fig. 7. One substructure, labeled Substructure $A$, includes the applied load while the other substructure, labeled Substructure $B$, contains the crack. Because the force in Substructure A is located at the center of the total beam, Substructure A was slightly longer than Substructure B. The displacement and force degrees of freedom associated with the external load used in the synthesis are the transverse displacement and the applied vertical force in Substructure A. For coupling between two substructures, the six translational displacement degrees of freedom at each end of the substructures were used. 


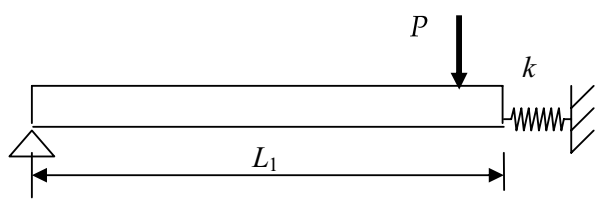

Substructure $A$

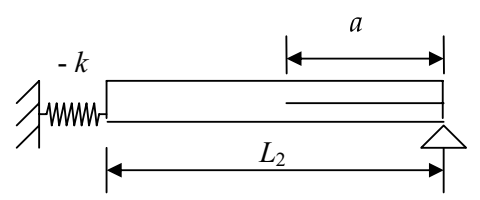

Substructure $B$

Fig. 7. Substructures of the simply supported beam of Fig. 6 .

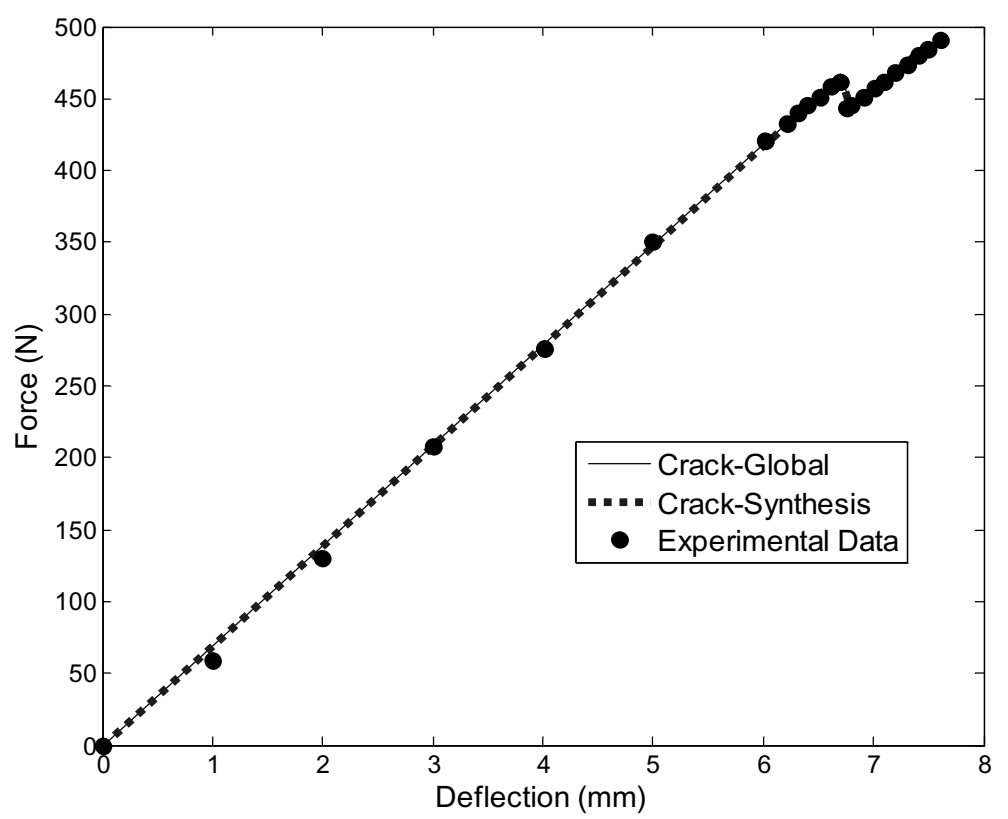

Fig. 8. Force vs. deflection curves at the center of a three point bending composite beam with a middle crack at one side.

Because each substructure has only one pin-joint support, there is still a rigid body mode in its stiffness matrix for static analysis, which prevents the calculation of the flexibility matrix, as needed by the synthesis. As a result, a spring-to-ground was attached to each substructure as shown in Fig. 7. The two springs had the same magnitude but one positive and the other negative. The locations of the springs were selected such that the spring effect was cancelled out when the substructures were coupled together. In other words, the springs were attached to two nodes, respectively, that would be directly coupled together.

The numerical result is compared to the experimental data as shown in Fig. 8. They agree very well. In the numerical model, crack growth was considered by checking the computed energy release rate against its critical value. When the former exceeded the latter, the crack advanced to the next element. Then, the new energy release rate was computed again and compared to the critical value. This process repeated until there was no more crack growth under a given deflection at the center of the beam. Once a stable crack size was determined, the force corresponding to the given deflection was computed. Then, another increment of the displacement was applied to the beam. This procedure continued so that the force-displacement curve was obtained as shown in Fig. 8. As the crack grew beyond a certain critical size, there was a significant load drop in the displacement-controlled situation. As explained, Fig. 9 illustrates the crack growth along with the applied displacement at the center of the beam. The figure shows that the crack did not propagate until the applied deflection reached greater than $6.6 \mathrm{~mm}$.

The same problem was also analyzed using the conventional finite element model without using the synthesis technique. This solution also agrees well with other solutions as seen in Fig. 8. However, the computational time was much more extensive for the traditional model without the synthesis technique. Figure 10 compares the execution time between two computer models with and without the synthesis technique using a PC. In other word, the ratio of 


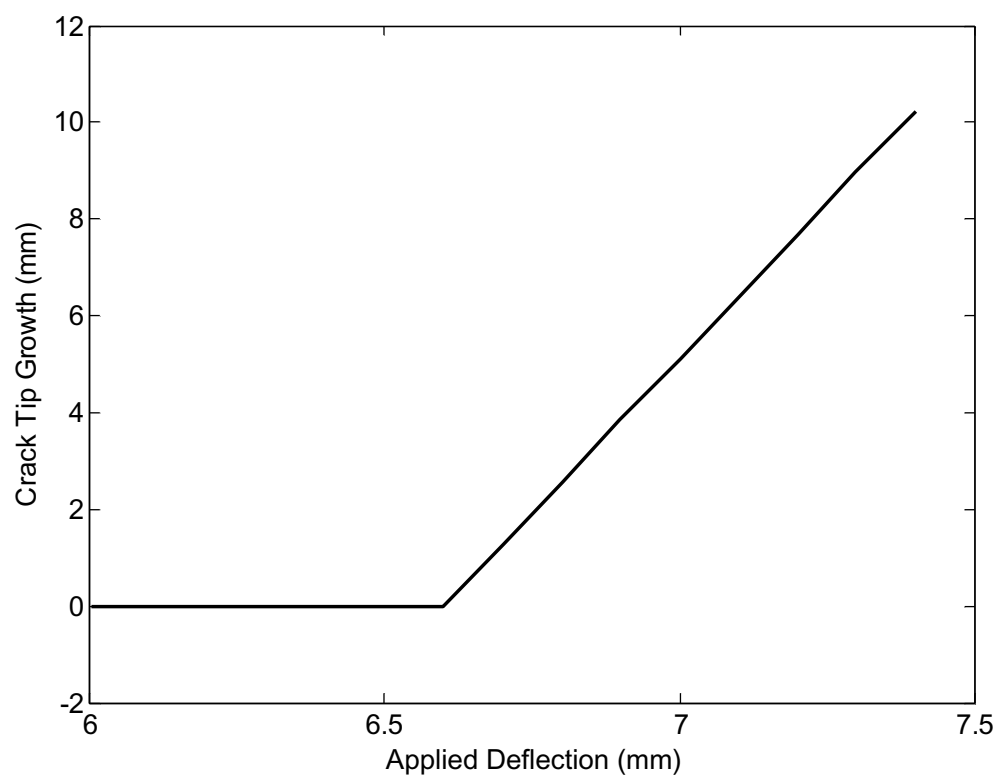

Fig. 9. Plot of crack growth as a function of the applied deflection at the center of a three point bending composite beam with a middle crack at one side.

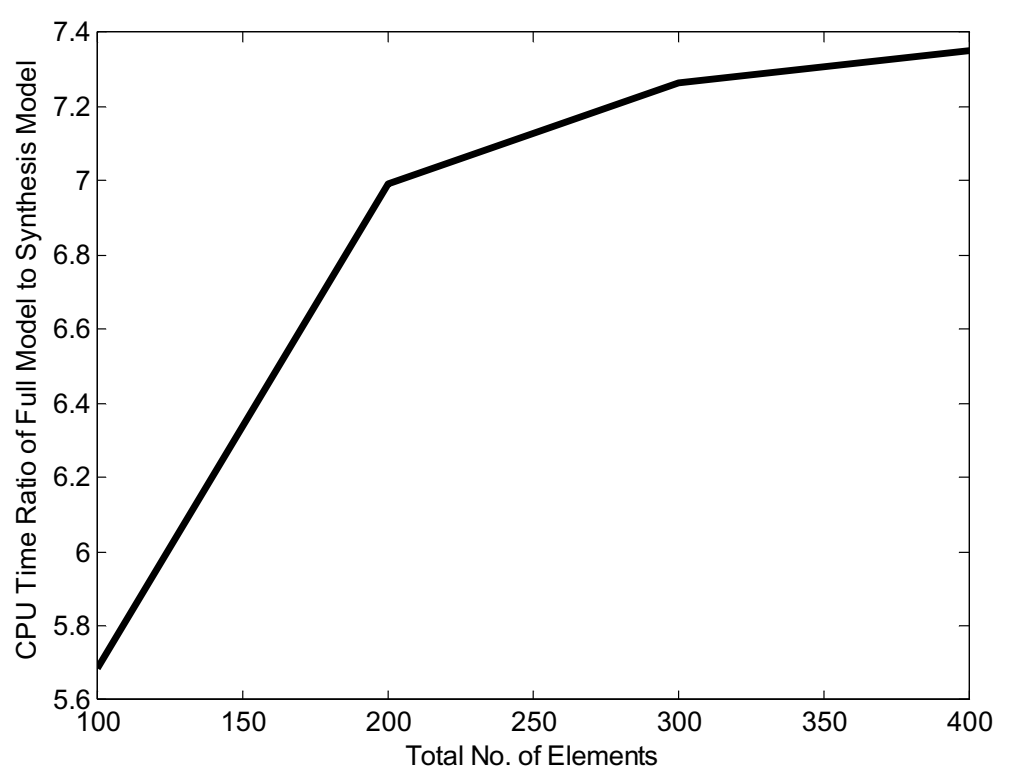

Fig. 10. Comparison of execution times between synthesis model and traditional full model as a function of the total number of elements in the model.

the CPU time without the synthesis technique divided by that with the synthesis technique was plotted as a function of the number of elements in the given problem. As expected, the synthesis technique proved more computationally efficient than the traditional full model. As the total number of elements in the model increased in the problem, the synthesis model became more efficient. Figure 10 suggests the CPU ratio curve approaches asymptotically to a limit value around 7.5. This characteristic behavior is problem-dependent.

If the number of degrees of freedom of a substructure which does not contain a change such as Substructure $A$ in Fig. 7 became larger compared to the degrees of freedom in the other substructure such as Substructure $B$ in 
Fig. 7, the computational savings associated with the substructuring synthesis technique increases further. For the present problem, both substructures had almost the same number of degrees of freedom. They only differed by 12. However, as the number of degrees of freedom in Substructure $A$ in Fig. 7 became double, the computational efficiency increased more than 15 times.

\section{Conclusions}

Frequency domain structural synthesis, functioning as a substructure coupling technique was applied to analyze quasi-static crack growth of an interface crack in a composite beam. This modeling of an interface crack behavior has been critical to predict the failure strength of scarf joints of composite structures. The synthesis technique contributed to reducing the computational time significantly as compared to the conventional full analysis technique. The actual computational saving associated with the substructuring synthesis technique depends on the number of degrees of freedom of a substructure which does not contain a change such as Substructure $A$ in Fig. 7 . If that number of degrees of freedom becomes larger, the computational efficiency becomes increases. This is frequently the case in real structures because cracks and damage are usually very localized in those structures.

In addition, the beam elements with displacement degrees of freedom only were useful to model a crack and its propagation. The predicted crack growth behavior agreed well with the experimental result in terms of the load-displacement curve. The extension of this work for subsequent studies includes quasi-static or transient crack growth in composite plate and shell structures in order to predict and monitor crack propagation in such structures.

\section{References}

[1] G. Kron, 1939, Tensor Analysis of Networks, John Wiley \& Sons, Inc., New York.

[2] B. Jetmundsen, R.L. Bielawa and W.G. Flannelly, Generalized Frequency Domain Substructure Synthesis, Journal of the American Helicopter Society 33(1) (1988), 55-64.

[3] J.H. Gordis and B. Neta, Fast Transient Analysis for Locally Nonlinear Structures by Recursive Block Convolution, ASME Journal of Vibration and Acoustics 123 (2001), 545-547.

[4] J.H. Gordis and J.L. Radwick, Efficient Transient Analysis for Large Locally Nonlinear Structures, Shock and Vibration 6 (1999), 1-9.

[5] J.H. Gordis, Integral Equation Formulation for Transient Structural Synthesis, AIAA Journal 33 (1995), $320-324$.

[6] J.H. Gordis, Structural Synthesis in the Frequency Domain: A General Formulation, Shock and Vibration 1 (1994), 461-471.

[7] J.H. Gordis, R.L. Bielawa and W.G. Flannelly, A General Theory for Frequency Domain Structural Synthesis, Journal of Sound and Vibration 150 (1991), 139-158.

[8] Y.W. Kwon, R. Slaff, S. Bartlett and T. Greene, Enhancement of Composite Scarf Joint Interface Strength through Carbon Nanotube Reinforcement, Journal of Materials Science 33 (2008), 6695-6703.

[9] J.H. Gordis and W.G. Flannelly, Analysis of Stress due to Fastener Tolerance in Assembled Components, AIAA Journal 32 (1994), 2440-2445.

[10] Y.W. Kwon, D. Salinas and M.J. Neibert, Thermally Induced Stresses in a Trilayered System, Journal of Thermal Stresses 17 (1994), 491-507.

[11] Y.W. Kwon and H. Bang, The Finite Element Method Using MATLAB, (2nd ed.), CRC Press, Boca Raton, Florida, 2000.

[12] M. Todo, T. Nakamura and K. Takahashi, Effects of Moisture Absorption on the Dynamic Interlaminar Fracture Toughness of Carbon/Epoxy Composites, Journal of Composite Materials 34 (2000), 630-648. 

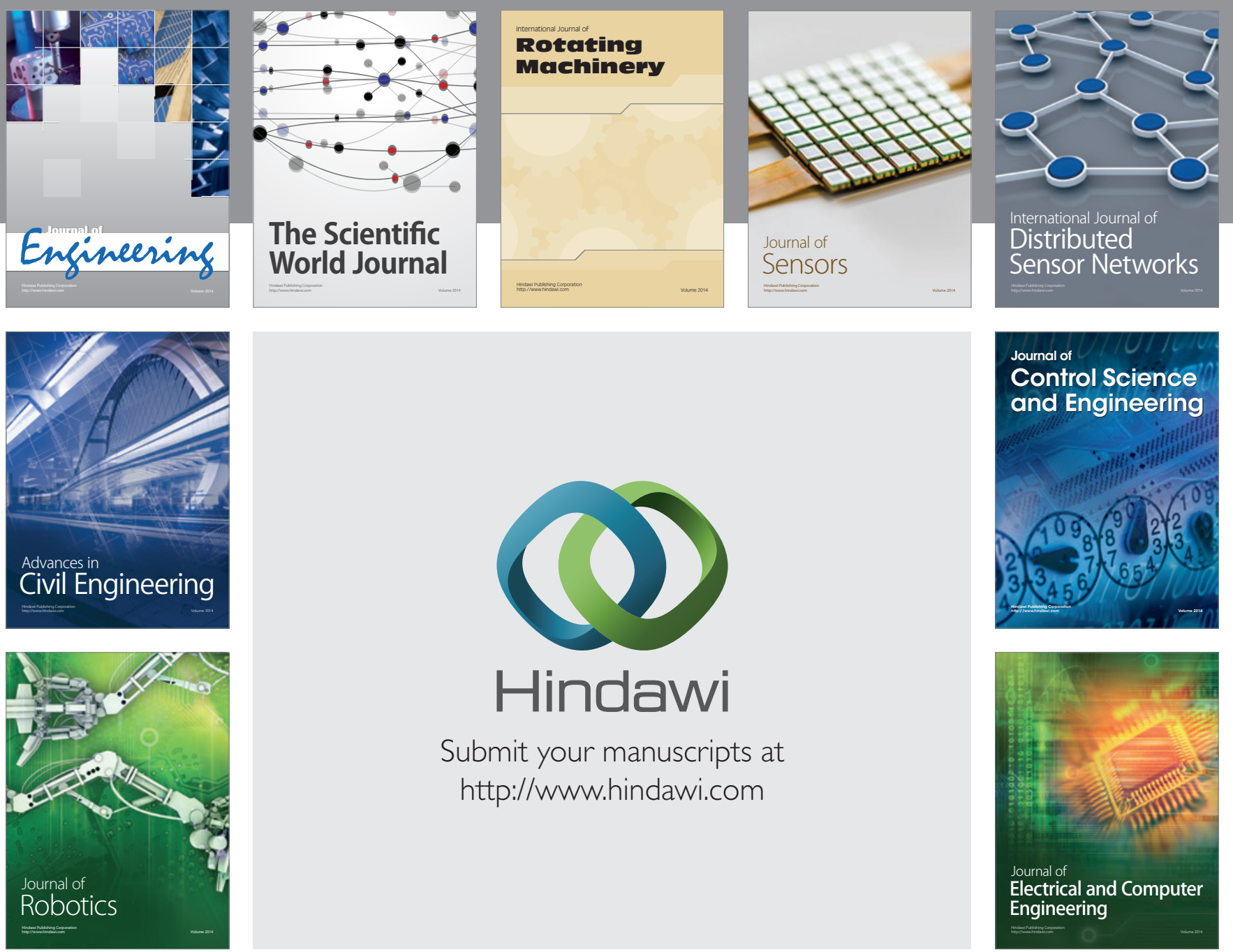

Submit your manuscripts at

http://www.hindawi.com
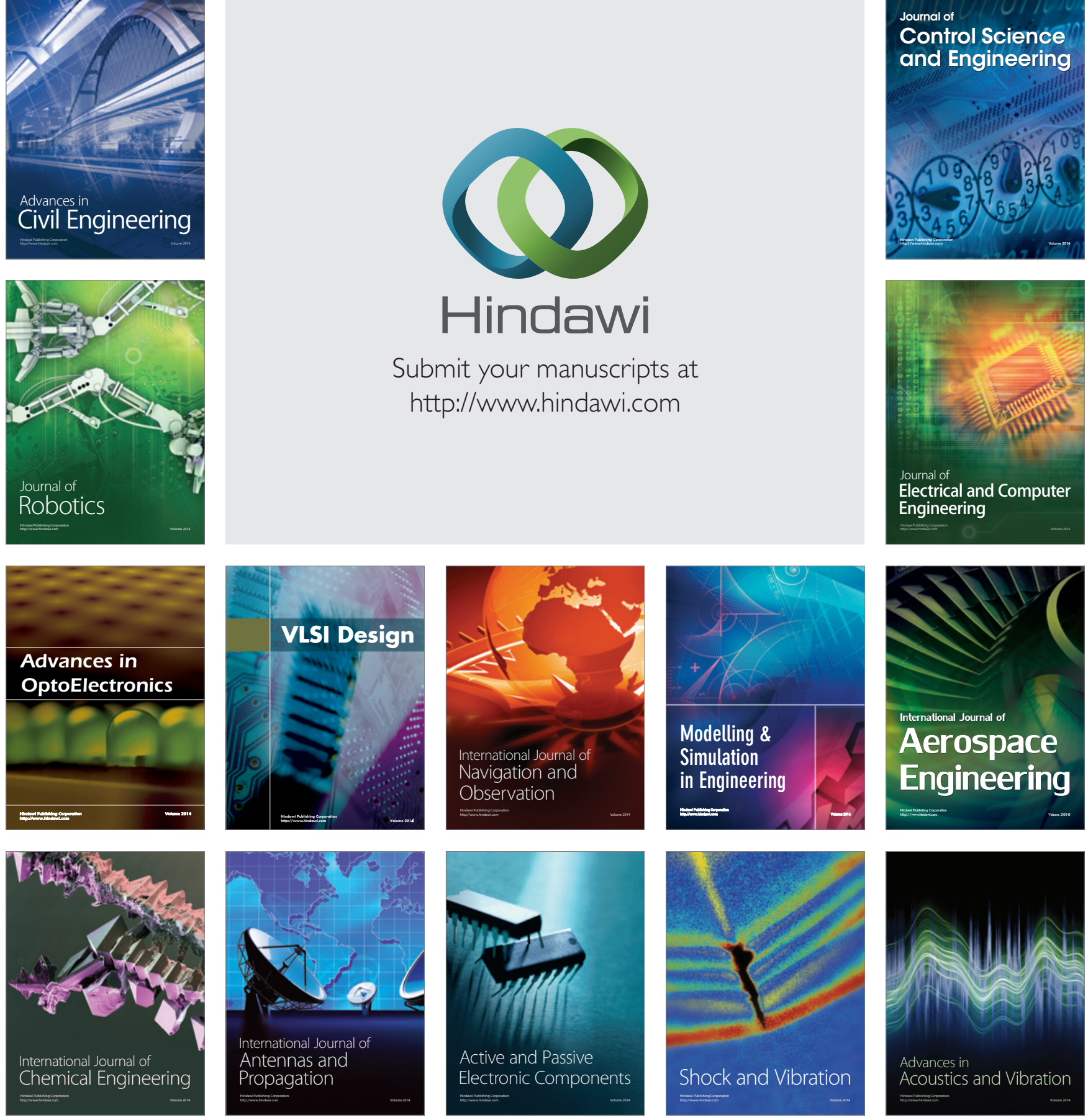